\title{
Decomposition of the transition phase in multi-sideband schemes for reconstruction of attosecond beating by interference of two-photon transitions
}

\author{
Divya Bharti $\odot,{ }^{1}$ David Atri-Schuller $\odot,{ }^{2}$ Gavin Menning $\odot,{ }^{2}$ Kathryn R. Hamilton $\odot,{ }^{2}$ Robert Moshammer, ${ }^{1}$ Thomas Pfeifer, ${ }^{1}$ \\ Nicolas Douguet, ${ }^{3}$ Klaus Bartschat $\odot{ }^{2},{ }^{2}$ and Anne Harth ${ }^{1, *}$ \\ ${ }^{1}$ Max-Planck-Institute for Nuclear Physics, D-69117 Heidelberg, Germany \\ ${ }^{2}$ Department of Physics and Astronomy, Drake University, Des Moines, Iowa 50311, USA \\ ${ }^{3}$ Department of Physics, Kennesaw State University, Marietta, Georgia 30060, USA
}

(Received 25 November 2020; accepted 2 February 2021; published 26 February 2021)

\begin{abstract}
Reconstruction of attosecond beating by interference of two-photon transitions (RABBITT) is a technique that can be used to determine the phases of atomic transition elements in photoionization processes. In the traditional RABBITT scheme, the so-called asymptotic approximation considers the measured phase as a sum of the Wigner phase linked to a single-photon ionization process and the continuum-continuum phase associated with further single-photon transitions in the continuum. In this paper, we extend the asymptotic approximation to multisideband RABBITT schemes. The predictions from this approximation are then compared with results obtained by an $a b$ initio calculation based on solving the time-dependent Schrödinger equation for atomic hydrogen.
\end{abstract}

DOI: 10.1103/PhysRevA.103.022834

\section{INTRODUCTION}

The reconstruction of attosecond beating by interference of two-photon transitions (RABBITT) technique was originally introduced for the temporal characterization of attosecond pulse trains (APTs) produced via high-order harmonic generation (HHG) [1]. The utility of this technique was later extended to measure the relative photoionization time delay from different valence shells in argon [2]. Nowadays, RABBITT is extensively employed to study the attosecond dynamics in atoms [2-5], molecules [6,7], and solids [8-10].

RABBITT is a pump-probe interferometric technique, in which an extreme ultraviolet (XUV) APT ionizes a target gas in the presence of a time-delayed near-infrared (NIR) pulse, and the kinetic-energy spectra of the photoelectrons are recorded as a function of the varied relative delay. Without the NIR probe photon, the photoelectron spectrum consists of discrete peaks (here also termed "harmonics") corresponding to the high-order harmonic peaks in the XUV spectrum. The presence of the probe field leads to the appearance of sidebands as additional peaks between the discrete harmonic peaks. In the traditional RABBITT scheme, only one sideband is formed between two consecutive harmonic peaks. This requires a minimum of one bound-continuum (bc) transition and one continuum-continuum (cc) transition. For RABBITT, two paths leading to the same sideband interfere. As the time

\footnotetext{
*anne.harth@mpi-hd.mpg.de
}

Published by the American Physical Society under the terms of the Creative Commons Attribution 4.0 International license. Further distribution of this work must maintain attribution to the author(s) and the published article's title, journal citation, and DOI. Open access publication funded by the Max Planck Society. delay $(\tau)$ between the pump and probe pulse is varied, the sideband signal is periodically modulated. The phase of this delay-dependent modulation is written as

$$
\Delta \phi=\Delta \phi_{\mathrm{XUV}}+\Delta \phi_{\text {atom }}
$$

where $\Delta \phi_{\mathrm{XUV}}$ is the phase related to the average group delay of the XUV pulses. In many cases, the atomic phase $\Delta \phi_{\text {atom }}$ can be conveniently split into two contributions:

$$
\Delta \phi_{\text {atom }}=\Delta \eta+\Delta \phi^{c c} \text {. }
$$

The Wigner-like phase shift $\Delta \eta$ originates from XUV-driven bc-transition processes, while the cc-transition phase $\Delta \phi^{c c}$ is associated with additional absorption and emission of a probe photon by the photoelectron. This relation is well accepted and was derived by Dahlström et al. [11] using the "asymptotic approximation," in which the asymptotic form of the scattering wave function is used to calculate the two-photon ionization amplitude. In this approximation, $\phi^{c c}$ becomes universal, as it depends on the charge of the residual ion, the photoelectron's kinetic energy, and the probe frequency, but not on the details of the atomic system. Dahlström et al. [11] formulated an analytical expression of $\phi^{c c}$, which is additionally independent of the angular momentum $\ell$.

However, the actual $\phi^{c c}$ does depend on the angular momenta [11-13], and this dependence becomes significant close to the ionization threshold. Since the $\ell$ dependence of $\phi^{c c}$ decreases with increasing photoelectron energy, we will generally neglect it in the discussion below, except for pointing out occasions where this dependence may become important.

For the one-sideband (1-SB) RABBITT setup, the probefield intensity is usually kept low to avoid significant contributions from multiple cc transitions. Increasing this intensity leads to the formation of higher-order sidebands, which then may overlap with the harmonic bands [14]. Lately, 
other forms of RABBITT schemes comprising more than one sideband between two consequent harmonic photoelectron peaks were also proposed and realized. For example, a two-sideband (2-SB) RABBITT configuration was used in an attosecond-pulse shaping measurement [15], and an experimental technique for a three-sideband (3-SB) RABBITT scheme was proposed to extract the cc-related phase separately by cancelling contributions from the Wigner phase [16].

In all these other schemes, multiple cc transitions are involved. However, a similar description and interpretation like Eq. (2) for multiphoton continuum transitions is by no means obvious. In this paper, we introduce a decomposition approximation by extending the asymptotic approximation to higher-order matrix elements as mentioned by Dahlström et al. [17].

This decomposition approximation leads to the interpretation that the final RABBITT phase is built up from the phases of stepwise transitions of the photoelectron, i.e., first the XUV-induced bc transition, and then subsequent $c c$ transitions, each involving a single IR photon.

This paper is structured as follows. In Sec. II, we outline the basic equations on which the paper is built. This is followed by Sec. III, where we first discuss the well-known 1-SB case before we introduce the decomposition approximation, which is then formally applied to the 3-SB case. Details of our derivation are given in the Appendix. Section IV provides a brief summary of the $a b$ initio numerical calculations, against which the predictions of the decomposition approximation are tested and discussed in Sec. V. We finish with a summary and an outlook regarding potential consequences for future experiments.

\section{GENERAL FORMULATION}

The RABBITT technique is based on the interference of different quantum paths leading to the same final energy. All equations in the present paper are written in the nonrelativistic single-active electron (SAE) picture, assuming that the initial bound electron has orbital angular momentum $\ell_{i}=0$ (often omitted in the notation below) and considering linearly polarized electric fields. Unless indicated otherwise, atomic units are used.

In the framework of time-dependent perturbation theory, the general expression of the transition amplitude describing a quantum path from an initial state $|i\rangle$ to a final state $\left|\vec{k}_{N}\right\rangle$ with asymptotic momentum $\vec{k}_{N}$, upon absorption of one XUVpump photon $(\Omega)$ and $(N-1)$ IR-probe photons $(\omega)$, is given by the $N$ th-order matrix element [18]

$$
M_{P}^{(N)}\left(\vec{k}_{N}\right)=-i \tilde{E}_{\Omega} \tilde{E}_{\omega}^{N-1}\left\langle\vec{k}_{N}\left|z \prod_{n=0}^{N-2}\left[G^{+}\left(\epsilon_{i}+\Omega+n \omega\right) z\right]\right| i\right\rangle,
$$

where $G^{+}$is the retarded resolvent of the free-field Hamiltonian, $\epsilon_{i}$ is the initial state energy, $z$ is the electric dipole operator, and $P$ specifies the path by which the final state is reached.

By projecting the final continuum states for the photoelectron on a partial-wave basis, the matrix elements corresponding to the different angular momentum channels can be disentangled as

$$
M_{P}^{(N)}\left(\vec{k}_{N}\right)=\tilde{E}_{\Omega} \tilde{E}_{\omega}^{N-1} \sum_{\ell_{N}} \mathcal{M}_{P, \ell_{N}}^{(N)}\left(k_{N}\right) Y_{\ell_{N}, 0}\left(\hat{k}_{N}\right) .
$$

The sum over $\ell_{N}$ represents the possible orbital angular momenta in the final state, and $Y_{\ell_{N}, 0}$ are spherical harmonics. Furthermore, $\tilde{E}_{\Omega}=E_{\Omega} \mathrm{e}^{i \phi_{\Omega}}$ and $\widetilde{E}_{\omega}=E_{\omega} \mathrm{e}^{i \omega \tau}$ (for absorption) are the complex electric-field amplitudes of the XUV-pump $(\Omega)$ and NIR-probe $(\omega)$ pulses, respectively.

Although much effort has been put into estimating multiphoton transition matrix elements [19,20], it remains challenging to accurately calculate the phases of multiphoton transition elements for a general target other than atomic hydrogen, even for $N=2$. Hence, finding a suitable approximation seems highly desirable.

\section{DECOMPOSITION OF THE RABBITT PHASE}

We start this section by applying the asymptotic approximation to a hydrogenic system to simplify the second-order matrix element $M^{(N=2)}$. We then extend the ideas behind the asymptotic approximation to higher-order matrix elements to arrive at a decomposition relation (see the Appendix for details) and subsequently apply it to the 3-SB RABBITT case.

\section{A. 1-SB RABBITT}

Figure 1(a) shows the energy-level diagram and transition pathways involved in a traditional (second-harmonic) 1-SB RABBITT scheme. To explain the appearance of the sideband $S$, one needs to consider only two paths, $\mathrm{A}$ and $\mathrm{B}$, which can both be described by second-order electric dipole transitions. Path A corresponds to the absorption of one XUV photon of energy $\Omega_{q+1}=(q+1) 2 \omega$ from the harmonic $H_{q+1}$ and emission of one probe $(2 \omega)$ photon, while path B corresponds to the absorption of an XUV photon of energy $\Omega_{q-1}=(q-1) 2 \omega$ from the lower harmonic $H_{q-1}$ and the absorption of a probe photon. Both paths lead to the same final continuum state $|f\rangle$ with asymptotic photoelectron momentum $k_{f}$, thus resulting in the sideband $S$. Using the notation of Eq. (4), the two-photon transition amplitudes for paths $\mathrm{A}$ and $\mathrm{B}$ are expressed as

$$
\begin{aligned}
& M_{A}^{(2, e)}\left(\vec{k}_{f}\right)=\tilde{E}_{2 \omega}^{*} \tilde{E}_{q+1} \sum_{\ell} \mathcal{M}_{A, \ell}^{(2, e)}\left(k_{f}\right) Y_{\ell, 0}\left(\hat{k}_{f}\right), \\
& M_{B}^{(2, a)}\left(\vec{k}_{f}\right)=\tilde{E}_{2 \omega} \tilde{E}_{q-1} \sum_{\ell} \mathcal{M}_{B, \ell}^{(2, a)}\left(k_{f}\right) Y_{\ell, 0}\left(\hat{k}_{f}\right) .
\end{aligned}
$$

The superscript $e(a)$ indicates the pathway where a probe photon $(2 \omega)$ is emitted (absorbed). $\ell\left(=\ell_{f}\right)$ is the angular momentum of the final state $(|f\rangle)$.

The angle-resolved sideband signal is formed as the coherent sum of all the quantum paths leading to the same final momentum state:

$$
S\left(\tau, \vec{k}_{f}\right) \propto\left|M_{A}^{(2, e)}\left(\vec{k}_{f}\right)+M_{B}^{(2, a)}\left(\vec{k}_{f}\right)\right|^{2} .
$$

The phase difference between the absorption and emission paths is varied by changing the time delay $(\tau)$ between the pump and the probe pulses. This results in an oscillation of the sideband signal as a function of the relative pulse delay. In 


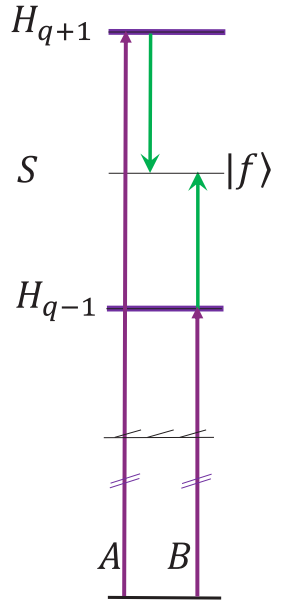

(a)

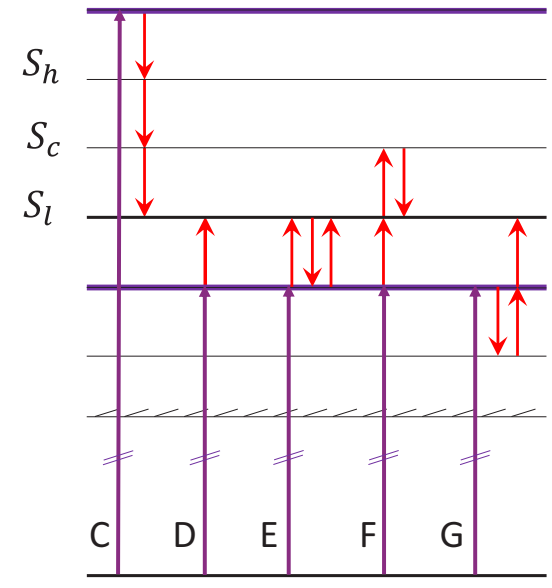

(b)
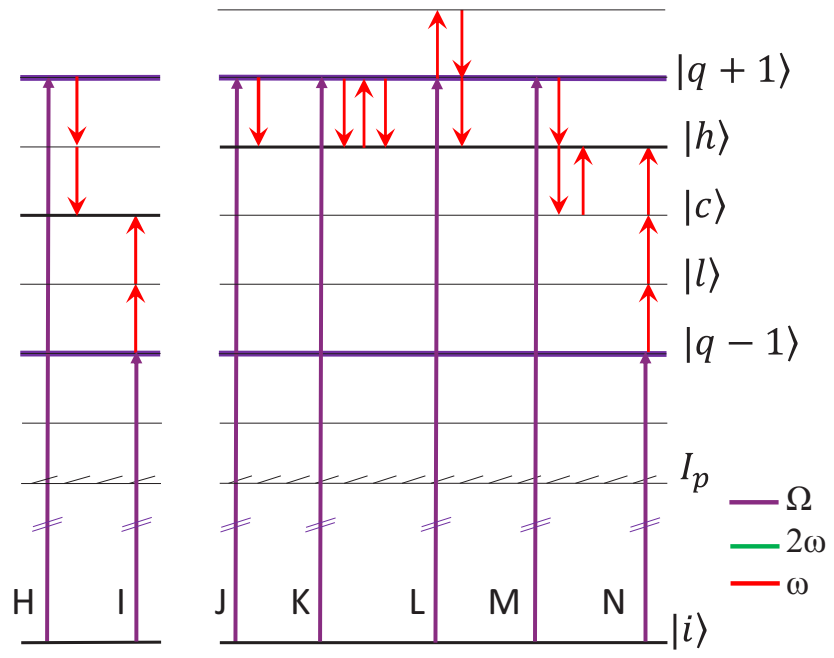

FIG. 1. Energy-level schemes in (a) traditional RABBITT and (b) 3-SB RABBITT. Photoionization by an XUV APT results in the appearance of harmonic peaks $H_{q-1}$ and $H_{q+1}$ (with $q$ being an even integer) in the photoelectron spectrum. The XUV spectrum $(\Omega)$ contains only odd harmonics of the $2 \omega$ pulse. Exchange of NIR probe photons of frequency $2 \omega$ or $\omega$ leads to the development of (a) a single sideband or (b) three sidebands, respectively. Interference among the multiple quantum paths leading to final states with the same energy gives rise to periodic oscillations in the sideband signal as a function of delay between pump and probe. Only the lowest-order paths required to explain the oscillations in the signal are shown. Paths in which the probe photon is absorbed before the pump photon are ignored.

the following, we discuss only angle-integrated cases, which results in Eq. (7) becoming an incoherent sum of partial-wave contributions, i.e.,

$$
\begin{aligned}
S\left(\tau, k_{f}\right) & \propto \sum_{\ell}\left|\tilde{E}_{q+1} \tilde{E}_{2 \omega}^{*} \mathcal{M}_{A, \ell}^{(2, e)}\left(k_{f}\right)+\tilde{E}_{q-1} \tilde{E}_{2 \omega} \mathcal{M}_{B, \ell}^{(2, a)}\left(k_{f}\right)\right|^{2} \\
& \propto I_{0}+I_{1} \cos \left(4 \omega \tau-\Delta \phi_{\mathrm{XUV}}-\Delta \phi_{\text {atom }}\right)
\end{aligned}
$$

where $\Delta \phi_{\mathrm{XUV}}=\left(\phi_{q+1}-\phi_{q-1}\right)$ is the phase difference between the harmonic fields $(q+1)$ and $(q-1)$, while

$$
\Delta \phi_{\text {atom }}=\arg \left[\sum_{\ell} \mathcal{M}_{A, \ell}^{(2, e)} \mathcal{M}_{B, \ell}^{*(2, a)}\right]
$$

is the atomic phase.

The atomic phase contains the combined effect of the ionizing XUV pump and the NIR probe. It is not obvious whether the two contributions can be separated to recover the pure (Wigner-only) photoionization delay. For the 1-SB RABBITT scheme, Dahlström et al. [11] showed that the atomic phase can be split into a single-photon ionization phase $(\eta)$ and the measurement-induced cc-transition phase $\left(\phi^{c c}\right)$ when using the asymptotic approximation. They also derived an analytical expression for $\phi^{c c}$ corresponding to the single-photon freefree transition of the photoelectron in the vicinity of the parent ion:

$$
\phi_{k, \kappa}^{c c}=\arg \left[\frac{(2 \kappa)^{i Z / \kappa}}{(2 k)^{i Z / k}} \frac{\Gamma[2+i Z(1 / \kappa-1 / k)]+\gamma(k, \kappa)}{(\kappa-k)^{i Z(1 / \kappa-1 / k)}}\right] .
$$

Here $\kappa$ is the wave number of the initial state in the continuum while $k$ is the wave number of the final photoelectron momentum after the exchange of an NIR photon, $Z$ is the remaining charge on the parent ion, and $\gamma$ denotes a longrange amplitude correction. Details can be found in Ref. [11].
It can be verified that $\phi_{k, \kappa}^{c c}=-\phi_{\kappa, k}^{c c}$; i.e., the absolute cc phase for absorption and emission between two given energy levels is identical.

Using the asymptotic approximation, the phase of the two-photon ionization amplitudes for any particular transition channel can be expanded as [11]

$$
\begin{aligned}
\arg \left[\mathcal{M}_{A, \ell}^{(2, e)}\right] & \approx-\frac{\ell_{q+1} \pi}{2}+\eta_{\ell_{q+1}}+\phi_{f, q+1}^{c c}, \\
\arg \left[\mathcal{M}_{B, \ell}^{(2, a)}\right] & \approx-\frac{\ell_{q-1} \pi}{2}+\eta_{\ell_{q-1}}+\phi_{f, q-1}^{c c} .
\end{aligned}
$$

The Wigner-like phase $\eta$ depends on the angular momentum $\left(\ell_{q+1}\right.$ or $\left.\ell_{q-1}\right)$ and the energy $\left(\epsilon_{q+1}\right.$ or $\left.\epsilon_{q-1}\right)$ of the intermediate state reached upon the XUV absorption, while $\phi^{c c}$ depends only on the energy of the two states involved in the free-free transitions. In special cases, where only one angular momentum channel $\left(\ell_{q+1}=\ell_{q-1}=\lambda\right)$ is significant in the bc transition, the atomic phase shift can be written as

$$
\Delta \phi_{\text {atom }}=\arg \left[\sum_{\ell} \mathcal{M}_{A, \ell}^{(2, e)} \mathcal{M}_{B, \ell}^{*(2, a)}\right] \approx \Delta \eta_{\lambda}+\Delta \phi^{c c}
$$

with $\Delta \eta_{\lambda}=\eta_{\lambda}\left(\epsilon_{q+1}\right)-\eta_{\lambda}\left(\epsilon_{q-1}\right)$ and $\Delta \phi^{c c}=\phi_{f, q+1}^{c c}-\phi_{f, q-1}^{c c}$ denoting the Wigner and cc phase differences, respectively.

Equation (13) is the same as Eq. (2) and is broadly used to measure the Wigner delay in various systems. We emphasize, however, that $\phi^{c c}$ here does not depend on the different angular momenta involved in the cc transitions, and the bc-transition step contains only one dominant angular momentum channel. In cases where several angular momentum channels are involved in the bc transition or $\phi^{c c}$ depends on the angular momenta of the final states, the phase extracted from RABBITT experiments depends on the detection angle 
of the observed electron. The atomic phase $\Delta \phi_{\text {atom }}$ measured in angle-integrated RABBITT schemes then becomes the weighted average of the Wigner and cc phases of each contributing channel.

\section{B. Decomposition approximation}

For RABBITT schemes involving more than two photon transitions $(N>2)$, the calculation of the necessary matrix elements becomes an increasingly formidable task. To get around this difficulty, we apply the ideas of the asymptotic approximation to estimate the phases of the higher-order matrix elements. Details of our derivation are provided in the Appendix. This allows us to decompose the phase of higherorder matrix elements into a sum of the phases generated by several subsequent single-photon transitions:

$$
\begin{aligned}
\arg \left[\mathcal{M}_{P, \ell}^{(N)}\right] \approx & \frac{(N-2) \pi}{2}-\frac{\lambda \pi}{2}+\eta_{\lambda}+\phi_{k_{2}, k_{1}}^{c c}+\phi_{k_{3}, k_{2}}^{c c} \\
& +\cdots+\phi_{k_{N-2}, k_{N-1}}^{c c}+\phi_{k_{N}, k_{N-1}}^{c c} .
\end{aligned}
$$

The decomposition approximation can be interpreted as a stepwise buildup of the final phase, starting with promotion from the initial bound state to a continuum state by the XUV and followed by $N-1$ transitions within the continuum states driven by the NIR. We emphasize that this approximation requires that we only have one bc channel $(\lambda)$ and $\phi^{c c}$ does not depend on $\ell$.

\section{3-SB RABBITT}

As an example, we now apply the decomposition approximation to the 3-SB RABBITT case. In this scheme, the consecutive harmonic peaks in the photoelectron spectrum are separated by four times the probe photon energy $(\omega)$. Figure 1(b) shows the 3-SB RABBITT scheme and the dominant transition paths, up to fourth order, that are involved in the formation of three sidebands. The population of the center sideband $S_{c}$ requires the absorption of an XUV pump photon $(\Omega)$ and the exchange of at least two probe photons. There are two dominant paths (H and I) leading to $S_{c}$, which can both be described using third-order matrix elements.

On the other hand, to explain the oscillations of the lower sideband $S_{l}$ and the higher sideband $S_{h}$, one needs to consider fourth-order dipole transitions, since at least one such highorder process has to be involved (e.g., path C for $S_{l}$ and path $\mathrm{N}$ for $S_{h}$ ). Altogether, there are five transition terms involved: one second-order and four fourth-order terms. Interference among the paths $\mathrm{D}$ to $\mathrm{G}$, or similarly $\mathrm{J}$ to $\mathrm{M}$, however, does not result in delay-dependent oscillations. Furthermore, the relation $\phi_{k, \kappa}^{c c}=-\phi_{\kappa, k}^{c c}$, along with the decomposition approximation, implies that the phases from back and forth transitions between the same two energy levels will cancel out. Hence, apart from a trivial additional $\pi$ shift, the phases in all fourthorder absorption paths (E, F, and G) would be the same as in the second-order absorption path (D). Similarly, there would be no phase difference between the paths J, K, L, and $\mathrm{M}$. Within this approximation, therefore, we can ignore the higher-order paths $\mathrm{E}$ to $\mathrm{G}$ and $\mathrm{K}$ to $\mathrm{M}$, as they will only change the amplitude but not the phase of the oscillation. This results in the following equations:

$$
\begin{aligned}
S_{l}\left(\tau, k_{l}\right) & \propto \sum_{\ell}\left|\tilde{E}_{q+1} \tilde{E}_{\omega}^{* 3} \mathcal{M}_{C, \ell}^{(4, e)}\left(k_{l}\right)+\tilde{E}_{q-1} \tilde{E}_{\omega} \mathcal{M}_{D, \ell}^{(2, a)}\left(k_{l}\right)\right|^{2} \\
& =I_{0}^{l}+I_{1}^{l} \cos \left(4 \omega \tau-\Delta \phi_{\mathrm{XUV}}-\Delta \phi_{\mathrm{atom}}^{l}\right), \\
S_{c}\left(\tau, k_{c}\right) & \propto \sum_{\ell}\left|\tilde{E}_{q+1} \tilde{E}_{\omega}^{* 2} \mathcal{M}_{H, \ell}^{(3, e)}\left(k_{c}\right)+\tilde{E}_{q-1} \tilde{E}_{\omega}^{2} \mathcal{M}_{I, \ell}^{(3, a)}\left(k_{c}\right)\right|^{2} \\
& =I_{0}^{c}+I_{1}^{c} \cos \left(4 \omega \tau-\Delta \phi_{\mathrm{XUV}}-\Delta \phi_{\mathrm{atom}}^{c}\right), \\
S_{h}\left(\tau, k_{h}\right) & \propto \sum_{\ell}\left|\tilde{E}_{q+1} \tilde{E}_{\omega}^{*} \mathcal{M}_{J, \ell}^{(2, e)}\left(k_{h}\right)+\tilde{E}_{q-1} \tilde{E}_{\omega}^{3} \mathcal{M}_{N, \ell}^{(4, a)}\left(k_{h}\right)\right|^{2} \\
& =I_{0}^{l}+I_{1}^{l} \cos \left(4 \omega \tau-\Delta \phi_{\mathrm{XUV}}-\Delta \phi_{\mathrm{atom}}^{h}\right) .
\end{aligned}
$$

By applying the decomposition approximation to the various atomic phase contributions in the above equations, and again assuming that there is only one partial wave $(\lambda)$ created in the XUV ionization process, these phases can be written as

$$
\begin{aligned}
& \Delta \phi_{\text {atom }}^{l}= \arg \left[\sum_{\ell} \mathcal{M}_{C, \ell}^{(4, e)} \mathcal{M}_{D, \ell}^{*(2, a)}\right] \\
& \approx \Delta \eta_{\lambda}+\phi_{h, q+1}^{c c}+\phi_{c, h}^{c c}+\phi_{l, c}^{c c}-\phi_{l, q-1}^{c c}+\pi, \\
& \Delta \phi_{\text {atom }}^{c}=\arg \left[\sum_{\ell} \mathcal{M}_{H, \ell}^{(3, e)} \mathcal{M}_{I, \ell}^{*(3, a)}\right] \\
& \approx \Delta \eta_{\lambda}+\phi_{h, q+1}^{c c}+\phi_{c, h}^{c c}-\phi_{c, l}^{c c}-\phi_{l, q-1}^{c c}, \\
& \Delta \phi_{\text {atom }}^{h}=\arg \left[\sum_{\ell} \mathcal{M}_{J, \ell}^{(2, e)} \mathcal{M}_{N, \ell}^{*(4, a)}\right] \\
& \approx \Delta \eta_{\lambda}+\phi_{h, q+1}^{c c}-\phi_{h, c}^{c c}-\phi_{c, l}^{c c}-\phi_{l, q-1}^{c c}-\pi .
\end{aligned}
$$

Inserting the relation $\phi_{k, \kappa}^{c c}=-\phi_{\kappa, k}^{c c}$ into Eqs. (18)-(20), we see that the atomic phases in all three sidebands are the same, except for an additional phase of $\pi$ due to the fact that the higher and lower sidebands are created, respectively, by interference of two- and four-photon transitions, while the center sideband is created by two three-photon transitions. Note, however, that the final kinetic energies of the photoelectrons in the three sidebands are different.

\section{NUMERICAL CALCULATIONS}

In order to test the validity of the decomposition approximation, we performed $a b$ initio calculations on atomic hydrogen to examine the delay-dependent variation in the sideband signals for the 3-SB RABBITT scheme. We chose a pump pulse containing eight odd harmonics $(5,7,9, \ldots, 19)$ of the generating 400-nm pulse. The pulse duration of each single harmonic $\left(E_{q-1}\right)$ is $20 \mathrm{fs}$ and the peak intensity is $10^{9} \mathrm{~W} / \mathrm{cm}^{2}$. All harmonics are in phase, i.e., $\Delta \phi_{\mathrm{XUV}}=0$. The center wavelength of the probe pulse is $800 \mathrm{~nm}$, the pulse duration is also $20 \mathrm{fs}$, and the peak intensity is $10^{11} \mathrm{~W} / \mathrm{cm}^{2}$.

The calculations were performed with a further improved version of the computer code described by Douguet et al. [21]. We performed extensive checks to ensure convergence of the predictions with the number of partial waves included, independence of the results from both the radial and the time 


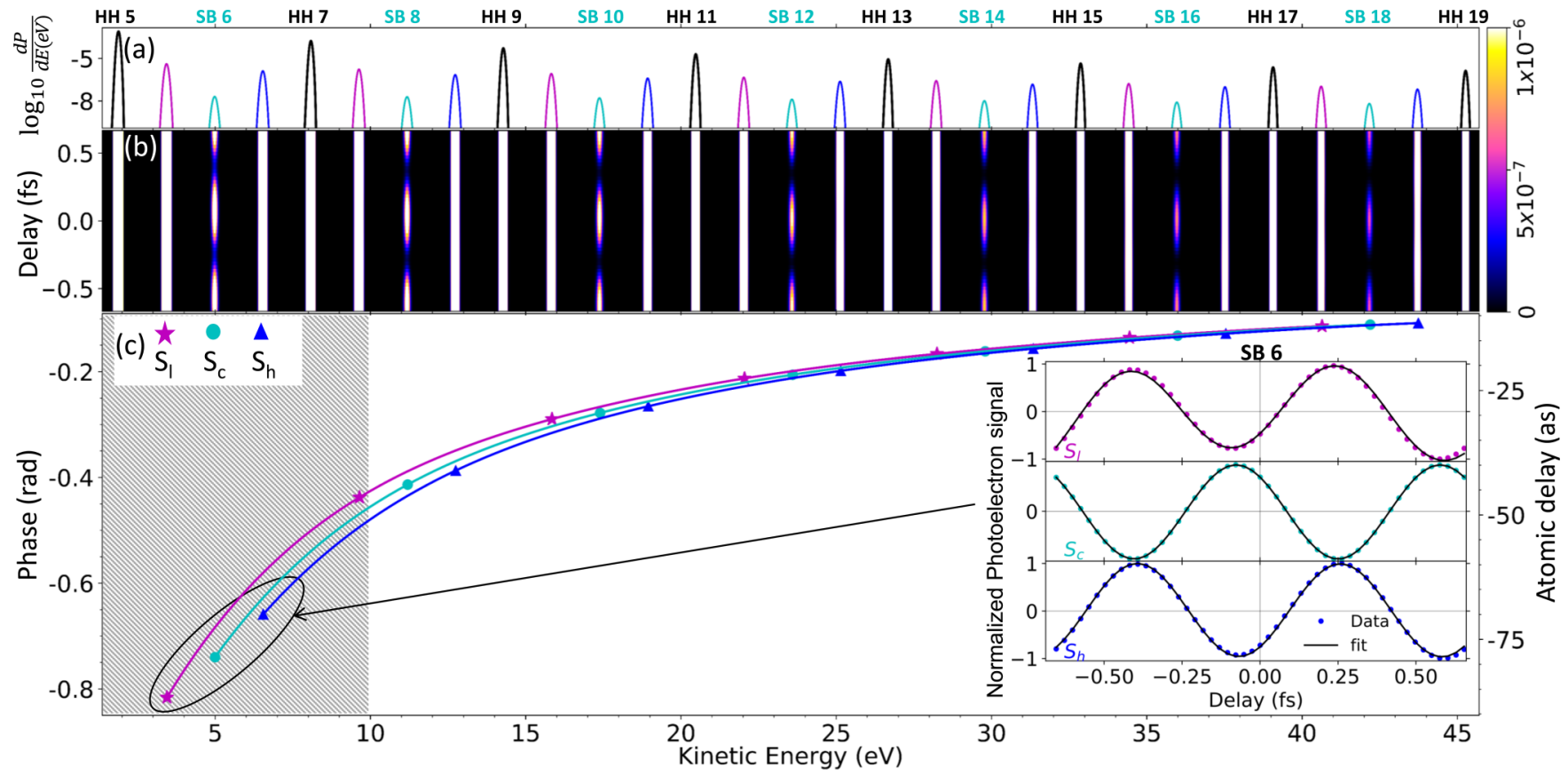

FIG. 2. (a) Photoelectron spectrum at maximum overlap between pump and probe ( $\tau=0$ ). (b) Contour plot of a 3-SB RABBITT spectrum. (c) Retrieved phases from the sideband oscillations. Inset: Fit of a cosine function (black line) to the photoelectron signals (dots) shown for the sixth sideband group order. After retrieving the sideband phases (procedure demonstrated in the inset), the additional $\pi$ shift was removed from $S_{l}$ and $S_{h}$ in (c) for clarity of presentation. $\Delta \phi_{\mathrm{XUV}}=0$ in this case. The conversion from phase (left axis) to time (right axis) is time $=$ phase $/(4 \omega)$.

steps in the discretization, and excellent agreement between the results obtained in either the length or the velocity form of the electric dipole operator. Given these rigorous tests, we are confident that the numerical predictions are highly accurate for this nonrelativistic one-electron problem and hence can be used to draw reliable conclusions about the validity (or the lack thereof) of the approximations outlined above.

\section{RESULTS AND DISCUSSION}

Figure 2(a) exhibits the photoelectron spectrum at zero time delay $(\tau=0)$ on a base-10 logarithmic scale. The pumpprobe delay-resolved RABBITT scan is shown in Fig. 2(b). Since the color bar was set to make the intensity oscillations in the center sideband $\left(S_{c}\right)$ visible, the other sideband oscillations are saturated with this setting. The inset in Fig. 2(c) shows the normalized data and the fits of the oscillatory part for the three sidebands in the lowest SB group. Note that the phase of the center sideband is $\pi$ out of phase as shown by Eq. (19). All the phases were retrieved from the data set shown in Fig. 2(b). For the data analysis, the sideband signals were integrated over an energy window of $0.25 \mathrm{eV}$ around the peaks. A constant component was subtracted from the integrated sideband signals and then renormalized. To retrieve the phase information, the data were then fit to a function containing a cosine term and a quadratic term to account for the decay of the signal with the delay (see the inset in Fig. 2). To simplify the comparison, the additional $\pi$ phases in $S_{l}$ and $S_{h}$ were removed.

As expected, the contrast of the oscillation is best in the center sideband, because both pathways, $\mathrm{H}$ and I, contribute to its population at the same (third) order. Since the modulations in the lower and higher sidebands originate from the interference of second-order and fourth-order transition terms, the depth of the corresponding oscillations is shallow before renormalization. In Fig. 2(c), the phases of the sidebands at their respective kinetic energies are plotted.

Figure 3 shows the phase of the center sideband and the corresponding analytical phase, as expressed in Eq. (19). The

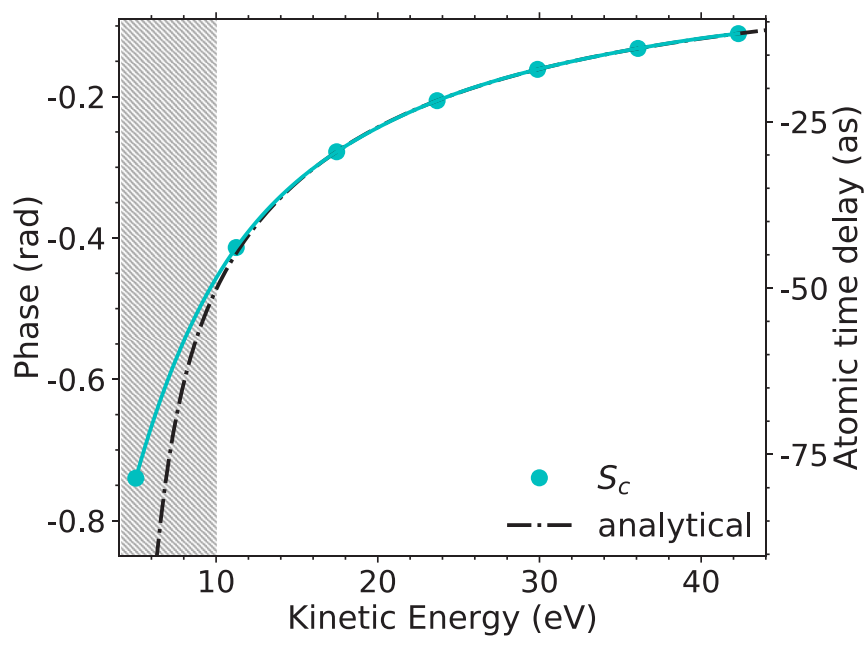

FIG. 3. Phase of the $S_{c}$ sideband (solid circles) extracted from the time-dependent Schrödinger equation calculation (see Fig. 2) and the corresponding analytical phase (dot-dashed line) estimated from Eq. (19). The difference above $12 \mathrm{eV}$ between the two curves corresponds to a time delay smaller than 0.01 as. 


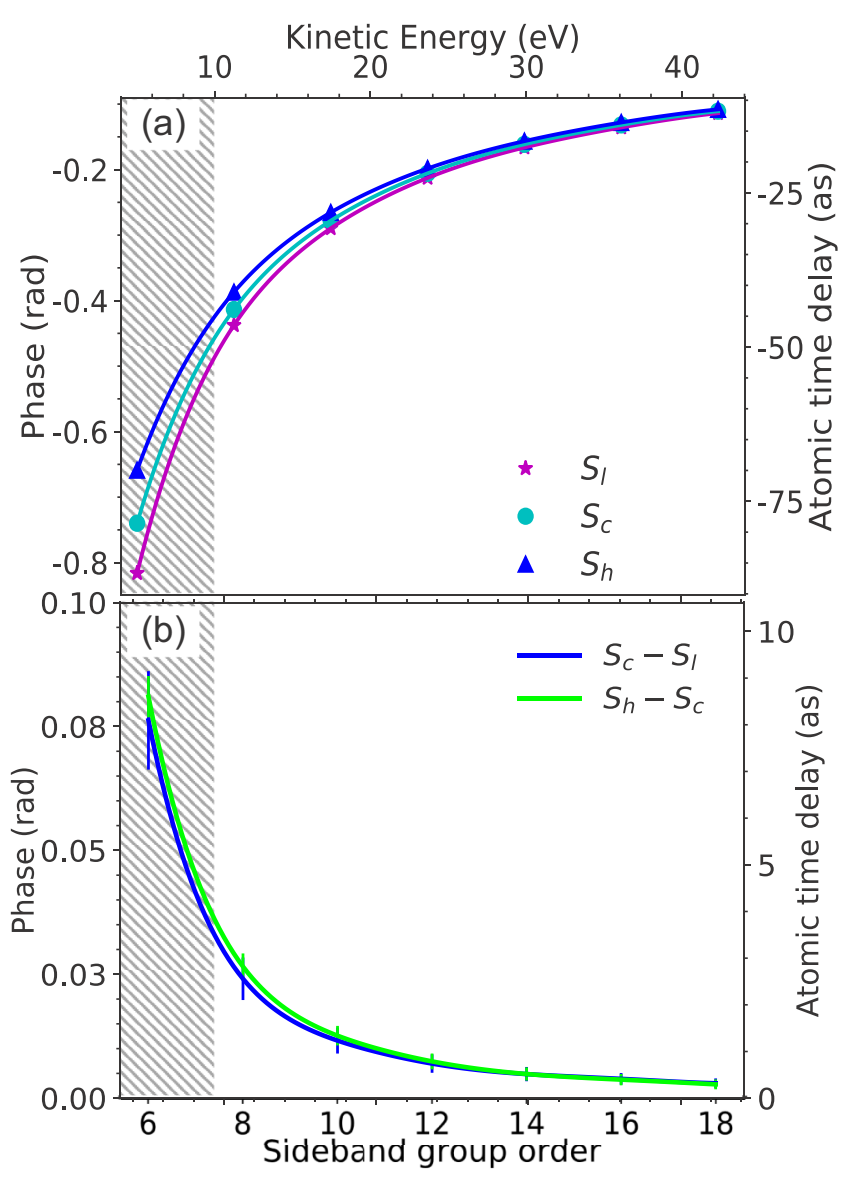

FIG. 4. (a) Phases of three sidebands, $S_{c}$ (dots), $S_{l}$ (stars), and $S_{h}$ (triangles), over the sideband group order. (b) Difference in the phases of nearby sidebands with respect to the center sideband. The error bar corresponds to the fitting error. The kinetic energy on the top horizontal axis corresponds to the kinetic energy of the center sideband.

single-photon ionization phase $\eta_{\lambda}$ for a hydrogenic system is simply the Coulomb phase:

$$
\eta_{\lambda}(\kappa, Z)=\arg \left[\Gamma\left(\lambda+1-i \frac{Z}{\kappa}\right)\right] .
$$

Here, $\kappa$ is the momentum of the released photoelectron, while $\lambda$ is the orbital angular momentum quantum number. In the case of atomic hydrogen, there is only one transition channel available by XUV ionization, which is $s \rightarrow p$, i.e., $\lambda=1$. The single-photon cc-phase contributions $\phi^{c c}$ for each transition are calculated using Eq. (10). For more details, see Ref. [11]. By inserting the expressions for $\eta$ and $\phi^{c c}$ into Eq. (19), the analytical phase associated with the center sideband $S_{c}$ is obtained.

The agreement between the two curves is remarkable for photoelectrons with a kinetic energy above $10 \mathrm{eV}$. Near threshold, however, the curves diverge. It should be mentioned that the analytical formula for $\phi^{c c}$ breaks down at low kinetic energies. Nevertheless, the good agreement between the analytical phases and the phase retrieved from the numerical calculation indicates that the decomposition approximation

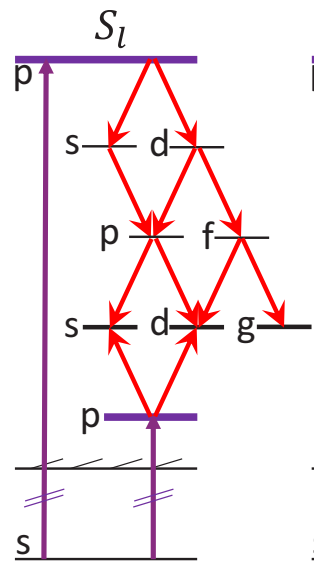

(a)

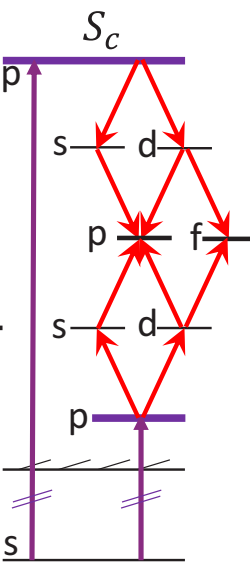

(b)

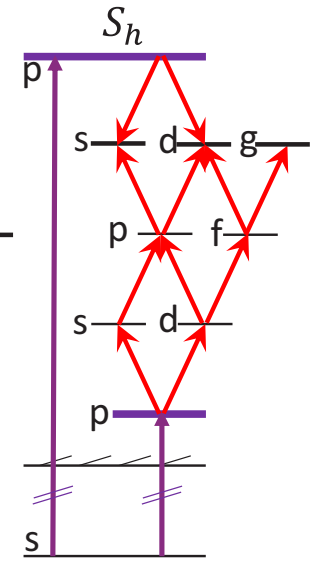

(c)
FIG. 5. Angular-momentum-resolved quantum paths leading to (a) the lower sideband, (b) the center sideband, and (c) the higher sideband. The angular momentum state $g$ does not contribute to the delay-dependent modulation of the angle-integrated sideband intensity. Only the dominant paths from Fig. 1 are shown.

works very well for the center sideband in a 3-SB RABBITT scheme.

Figure 2(c) is plotted again in Fig. 4(a), but now over the same sideband group order. We immediately see that the phases in the neighboring sidebands are not identical, but the difference between the phases obtained from the three sidebands steadily decreases with increasing kinetic energy. The phase difference between $S_{h}$ and $S_{l}$ in the same sideband group corresponds to a time delay of less than 6 as slightly above a photoelectron energy of $10 \mathrm{eV}$, but reduces to less than 2 as beyond $20 \mathrm{eV}$.

The fact that the phases in the three sidebands (Fig. 4) are not exactly the same could indicate that the decomposition approximation is not valid. However, we know from Fig. 3 that the approximation works well for the center sideband. Therefore, we now discuss the possible origins of the discrepancy, which is clearly visible for the $S_{h}$ and $S_{l}$ sidebands.

As mentioned in the Introduction, it has been shown that the actual $\phi^{c c}$ depends on the angular momentum of the states involved in the transitions [11-13]. In that case, back-andforth transitions between two energy levels in the continuum do not cancel out the cc phases, i.e., $\phi_{k, \kappa}^{c c}\left(\ell_{k}\right) \neq-\phi_{\kappa, k}^{c c}\left(\ell_{\kappa}\right)$, because there might be different channels involved in the two processes. As a consequence, the second-order absorption path (D) and the previously neglected fourth-order paths (E, F, and G) may not have the same phase. Similarly, the dependence of $\phi^{c c}$ on the angular momentum may result in a phase difference between the paths J, K, L, and M even when the decomposition approximation holds.

The angular momentum dependence of $\phi^{c c}$ may also manifest itself when one considers only the dominant paths contributing to the modulation of all sidebands (see Fig. 5). If an electron with $\ell=0$ starts in the ground state, the final wave function of the center sideband photoelectron after interaction with three photons will contain orbital angular quantum numbers $\ell=1$ and $\ell=3$. On the other hand, the lower (higher) sideband includes quantum numbers $\ell=0,2,4$ in 
the emission (absorption) path, and $\ell=0,2$ in the absorption (emission) path. Keeping this in mind, all the $\phi^{c c}$ corresponding to the same energy levels could be different, and hence the phases in the intermediate sidebands may not be the same.

We conclude that the phase difference between the three sidebands comes most likely from the neglected $\ell$ dependence of $\phi^{c c}$ while the applied decomposition approximation is reasonable. The good agreement between the $\ell$-independent analytical phase and the retrieved phases from the timedependent Schrödinger equation calculation for the $S_{c}$ (cf. Fig. 4) can be explained by considering the fact that both the emission and absorption paths are of the same order and contain the same set of possible partial waves ( $p$ and $f$ ). As hinted in Refs. [11,12] for 1-SB RABBITT calculations on He and $\mathrm{H}$, the absolute phase difference between distinct partial waves for the absorption paths is the same as for the emission paths. Hence the difference between the two paths nullifies the effect of the $\ell$ dependence. This is not true for the lower and higher sidebands, where the dominant interfering paths are of different orders.

However, while the angular-momentum dependence is particularly prominent for low kinetic energies of the photoelectron, it becomes increasingly negligible with growing kinetic energy [13]. This parallels our observation of improved agreement between the phases of all three sidebands in our 3-SB RABBITT scheme. Consequently, for sufficiently large photoelectron energies, where the angular momentum dependence of $\phi^{c c}$ is small, our calculations support the decomposition approximation to interpret the measured atomic phase in multi-sideband RABBITT schemes as stepwise onephoton transitions.

\section{SUMMARY AND OUTLOOK}

We studied the formation of sidebands and their oscillations in the 3-SB RABBITT scheme. The phases retrieved from the oscillation of the three sidebands contain the phases of higher-order dipole matrix elements, which are difficult to interpret. A decomposition approximation was attempted to simplify the phase extraction of the higher-order matrix elements as the sum of the phases of sequential one-photon transitions. The decomposition approximation along with the assumption that $\phi^{c c}$ is independent of the orbital angular momenta involved predicts that the phases extracted from all sidebands between two consecutive harmonics are the same.

In order to check these assumptions, we performed $a b$ initio calculations for atomic hydrogen. While the phases in all sideband groups are not identical, the difference decreases with increasing kinetic energy. This difference is attributed to the dependence of $\phi^{c c}$ on the angular momentum and the involvement of different $\ell$ channels in the three sidebands. We, therefore, conclude that while the decomposition approximation is an appropriate assumption when describing a multi-sideband RABBITT scheme, the dependence of $\phi^{c c}$ on the orbital angular momenta cannot be neglected for lowenergy sidebands.

Multi-sideband RABBITT provides an opportunity to probe deeper into the continuum of the ionic species. The present benchmark studies are important for a planned exper- iment using an argon target, which is experimentally much more suitable than atomic hydrogen. We already started numerical calculations for this system and presented preliminary results at a recent conference [22]. Note, however, that having the XUV APT ionize the electron from the $3 p$ bound orbital leads to continuum $s$ and $d$ waves, thereby further complicating the interpretation of the RABBITT phase. Work is currently in progress to address these issues.

In summary, we believe that multi-SB RABBITT opens up a frontier in the study of transition phases in photoionization processes. Since many more questions remain, we hope that the present paper will stimulate further work in this area.

\section{ACKNOWLEDGMENTS}

This work was supported by the Deutsche Forschungsgemeinschaft under DFG-SPP-1840-HA-8399/2-1 and by the U.S. National Science Foundation under Grants No. OAC1834740 and No. PHY-1803844 (D.A.S., G.M., K.R.H., K.B.) and No. PHY-2012078 (N.D.), as well as the XSEDE supercomputer allocation Grant No. PHY-090031. The calculations were carried out on Stampede2 and Frontera at the Texas Advanced Computing Center in Austin, Bridges at the Pittsburgh Supercomputing Center, and Comet at the San Diego Supercomputer Center.

\section{APPENDIX}

In this Appendix, we derive Eq. (14) by generalizing the asymptotic approximation for the two-photon matrix element introduced by Dahlström et al. [11]. In order to set the stage and introduce our shorthand notation for the often lengthy expressions, we first repeat the key ideas of the above paper and then apply them to the third- and higher-order matrix elements.

We begin by explicitly writing Eq. (3) as

$$
\begin{aligned}
M^{(N)} & \left(\vec{k}_{N} ; \Omega, \omega\right) \\
= & -i \tilde{E}_{\Omega} \tilde{E}_{\omega}^{N-1} \lim _{\varepsilon \rightarrow 0^{+}} \int d^{3} \vec{k}_{N-1}^{\prime} \cdots \int d^{3} \vec{k}_{2}^{\prime} \\
& \times \int d^{3} \vec{k}_{1}^{\prime} \frac{\left\langle\vec{k}_{N}|z| \vec{k}_{N-1}^{\prime}\right\rangle \cdots\left\langle\vec{k}_{2}^{\prime}|z| \vec{k}_{1}^{\prime}\right\rangle\left\langle\vec{k}_{1}^{\prime}|z| i\right\rangle}{\left(\epsilon_{N-1}-\epsilon_{N-1}^{\prime}+i \varepsilon\right) \cdots\left(\epsilon_{1}-\epsilon_{1}^{\prime}+i \varepsilon\right)} .
\end{aligned}
$$

Here $\epsilon_{i}$ is the initial state energy and $\epsilon_{i}+\Omega+n \omega=\epsilon_{n+1}$. As in Ref. [11], we neglect all contributions from bound states, since they are expected to be small if the photon energy for the bc transition is sufficiently high.

The final and intermediate continuum states are decomposed into partial waves as

$$
\varphi_{\vec{k}}(\vec{r})=(8 \pi)^{3 / 2} \sum_{\ell, m} i^{\ell} e^{-i \eta_{\ell}(k)} Y_{\ell, m}^{*}(\hat{k}) Y_{\ell, m}(\hat{r}) R_{k, \ell}(r),
$$

where $\eta_{\ell}(k)$ denotes the scattering phases, $Y_{\ell, m}$ the spherical harmonics, and $R_{k, \ell}$ are the radial parts. Splitting the initial state into its radial and angular parts according to

$$
\varphi_{n_{i}, \ell_{i}, m_{i}}(\vec{r})=Y_{\ell_{i}, m_{i}}(\hat{r}) R_{n_{i}, \ell_{i}}(r),
$$


using $z=\sqrt{\frac{4 \pi}{3}} r Y_{1,0}(\hat{r})$, and inserting Eqs. (A2) and (A3) into Eq. (A1) yields for $N=2$

$$
\begin{aligned}
M^{(2)}\left(\vec{k}_{2} ; \Omega, \omega\right)= & -i \frac{4 \pi}{3}(8 \pi)^{3 / 2} \tilde{E}_{\Omega} \tilde{E}_{\omega} \\
& \times \sum_{\ell_{2}, m_{2}}(-i)^{\ell_{2}} e^{i \eta_{2}} Y_{\ell_{2}, m_{2}}\left(\hat{k}_{2}\right) \\
& \times \sum_{\ell_{1}, m_{1}}\left\langle Y_{\ell_{2}, m_{2}}\left|Y_{1,0}\right| Y_{\ell_{1}, m_{1}}\right\rangle\left\langle Y_{\ell_{1}, m_{1}}\left|Y_{1,0}\right| Y_{\ell_{i}, m_{i}}\right\rangle \\
& \times T_{\ell_{2}, \ell_{1}, \ell_{i}}^{(2)}\left(k_{2} ; \Omega, \omega\right) \\
= & \tilde{E}_{\Omega} \tilde{E}_{\omega} \sum_{\ell_{2}} \mathcal{M}_{\ell_{2}, m_{2}}^{(2)}\left(k_{2}\right) Y_{\ell_{2}}\left(\hat{k}_{2}\right) .
\end{aligned}
$$

This defines $\mathcal{M}_{\ell_{2}, m_{2}}^{(2)}\left(k_{2}\right)$ and simplifies to Eq. (4) of the main text for $\ell_{i}=m_{i}=0$ and linearly polarized light $\left(m_{2}=0\right)$.

Defining the first-order radial matrix element

$$
T_{\ell_{1}, \ell_{i}}^{(1)}\left(k_{1}^{\prime} ; \Omega\right)=\left\langle R_{k_{1}^{\prime}, \ell_{1}}|r| R_{n_{i}, \ell_{i}}\right\rangle
$$

allows us to write the second-order element as

$$
\begin{aligned}
& T_{\ell_{2}, \ell_{1}, \ell_{i}}^{(2)}\left(k_{2} ; \Omega, \omega\right) \\
& \quad=\lim _{\varepsilon \rightarrow 0^{+}} \int_{0}^{\infty} d \epsilon_{1}^{\prime} \frac{\left\langle R_{k_{2}, \ell_{2}}|r| R_{k_{1}^{\prime}, \ell_{1}}\right\rangle T_{\ell_{1}, \ell_{i}}^{(1)}\left(k_{1}^{\prime} ; \Omega\right)}{\epsilon_{i}+\Omega-\epsilon_{1}^{\prime}+i \varepsilon} .
\end{aligned}
$$

The first-order perturbed wave function [11] is defined as

$$
\begin{aligned}
\left|\rho_{k_{1}, \ell_{1}}\right\rangle= & \lim _{\varepsilon \rightarrow 0^{+}} \int_{0}^{\infty} d \epsilon_{1}^{\prime} \frac{T_{\ell_{1}, \ell_{i}}^{(1)}\left(k_{1}^{\prime} ; \Omega\right)\left|R_{k_{1}^{\prime}, \ell_{1}}\right\rangle}{\epsilon_{1}-\epsilon_{1}^{\prime}+i \varepsilon} \\
= & \mathcal{P} \int_{0}^{\infty} d \epsilon_{1}^{\prime} \frac{T_{\ell_{1}, \ell_{i}}^{(1)}\left(k_{1}^{\prime} ; \Omega\right)\left|R_{k_{1}^{\prime}, \ell_{1}}\right\rangle}{\epsilon_{1}-\epsilon_{1}^{\prime}} \\
& -i \pi T_{\ell_{1}, \ell_{i}}^{(1)}\left(k_{1} ; \Omega\right)\left|R_{k_{1}, \ell_{1}}\right\rangle,
\end{aligned}
$$

where $\mathcal{P}$ denotes the principal value.

The key in deriving an analytic expression for the contribution to the phase of the matrix element is to replace the radial functions of the intermediate and final continuum states by their asymptotic form

$$
\lim _{r \rightarrow \infty} R_{k, \ell}(r) \approx \frac{N_{k}}{r} \sin \left(k r+\phi_{k, \ell}(r)\right) .
$$

Here $N_{k} \approx \sqrt{2 / \pi k}\left(1-Z /\left(2 r k^{2}\right)\right)$ is the amplitude of the asymptotic wave for a long-range potential $(-Z / r)$, and the asymptotic phase is $\phi_{k, \ell}(r)=(Z / k) \ln (2 k r)+\eta_{\ell}(k)-\pi \ell / 2$. Using the same steps and approximations as outlined in Ref. [11], this leads to the approximate form

$$
\lim _{r \rightarrow \infty} \rho_{1}(r) \approx-\frac{\pi N_{1}}{r} T^{(1)}\left(k_{1}\right) e^{i\left(k_{1} r+\phi_{1}(r)\right)}
$$

for the perturbed wave function after further compressing the notation by defining $R_{k_{n}, \ell_{n}} \equiv R_{n},\left|\rho_{k_{1}, \ell_{1}}\right\rangle \equiv\left|\rho_{1}\right\rangle, N_{k_{n}} \equiv N_{n}$, $\phi_{k_{n}, \ell_{n}}(r) \equiv \phi_{n}(r), T_{\ell_{1}, \ell_{i}}^{(1)}\left(k_{1} ; \Omega\right) \equiv T^{(1)}\left(k_{1}\right)$, and $\eta_{\ell_{n}}\left(k_{n}\right) \equiv \eta_{n}$.

The second-order matrix element then becomes

$$
\begin{aligned}
T_{2}^{(2)}\left(k_{2}\right)= & \left\langle R_{2}|r| \rho_{1}\right\rangle \\
\approx & \frac{T^{(1)}\left(k_{1}\right)}{i \sqrt{k_{1} k_{2}}} \int_{0}^{\infty} d r\left(r-\frac{1}{2}\left(\frac{1}{k_{1}^{2}}+\frac{1}{k_{2}^{2}}\right)\right) \\
& \times \sin \left(k_{2} r+\phi_{2}(r)\right) e^{i\left(k_{1} r+\phi_{1}(r)\right)} .
\end{aligned}
$$

After writing the sin term in exponential form, dropping the fast-oscillating term containing $k_{1}+k_{2}$ while keeping the term with $k_{1}-k_{2}$, and introducing the substitution variable $-i\left(k_{1}-k_{2}\right) r$, one obtains a $\Gamma$ function with complex argument. Using this function, we find

$$
\begin{aligned}
T_{2}^{(2)}\left(k_{2}\right) \approx & \frac{T^{(1)}\left(k_{1}\right)}{\sqrt{k_{1} k_{2}}} \frac{e^{-Z\left(1 / k_{1}-1 / k_{2}\right) \pi / 2}}{\left(k_{1}-k_{2}\right)^{2}} i^{\ell_{2}-\ell_{1}+1} e^{i\left(\eta_{1}-\eta_{2}\right)} \\
& \times \frac{\left(2 k_{1}\right)^{i Z / k_{1}}}{\left(2 k_{2}\right)^{i Z / k_{2}}} \frac{\left(\Gamma\left[2+i Z\left(1 / k_{1}-1 / k_{2}\right)\right]+\gamma\left(k_{2}, k_{1}\right)\right)}{\left(k_{1}-k_{2}\right)^{i Z\left(1 / k_{1}-1 / k_{2}\right)}}
\end{aligned}
$$

with $\quad \gamma\left(k_{2}, k_{1}\right)=\frac{i Z\left(k_{1}-k_{2}\right)}{2}\left(\frac{1}{k_{1}^{2}}+\frac{1}{k_{2}^{2}}\right) \Gamma\left[1+i Z\left(1 / k_{1}-1 / k_{2}\right)\right]$ accounting for the effect of the long-range potential [11]. The phase of the radial matrix element is

$$
\arg \left[T_{\ell_{2}, \ell_{1}, \ell_{i}}^{(2)}\left(k_{2}\right)\right]=\frac{\pi}{2}\left(\ell_{2}-\ell_{1}+1\right)+\left(\eta_{1}-\eta_{2}\right)+\phi_{2,1}^{c c},
$$

where

$$
\begin{aligned}
\phi_{2,1}^{c c} & \equiv \phi_{k_{2}, k_{1}}^{c c} \\
& =\arg \left[\frac{\left(2 k_{1}\right)^{i Z / k_{1}}}{\left(2 k_{2}\right)^{i Z / k_{2}}} \frac{\left(\Gamma\left[2+i Z\left(1 / k_{1}-1 / k_{2}\right)\right]+\gamma\left(k_{2}, k_{1}\right)\right)}{\left(k_{1}-k_{2}\right)^{i Z\left(1 / k_{1}-1 / k_{2}\right)}}\right],
\end{aligned}
$$

and we have used that $T_{\ell_{1}, \ell_{i}}^{(1)}\left(k_{1}\right)$ is real. Substituting Eq. (A11) back into Eq. (A4), the phase of the matrix element for the transition path $\ell_{i} \rightarrow \ell_{1} \rightarrow \ell_{2}$ is

$$
\begin{aligned}
\arg \left[\mathcal{M}_{\ell_{2}, \ell_{1}, \ell_{i}}^{(2)}\left(k_{2}\right)\right] \approx & \frac{-\pi}{2}-\frac{\pi \ell_{2}}{2}+\eta_{2}+\frac{\pi}{2}\left(\ell_{2}-\ell_{1}+1\right) \\
& +\eta_{1}-\eta_{2}+\phi_{2,1}^{c c}+T_{\ell_{1}, \ell_{i}}^{(1)}\left(k_{1}\right) \\
= & -\frac{\pi \ell_{1}}{2}+\eta_{1}+\phi_{2,1}^{c c} .
\end{aligned}
$$

Note the cancellations in this formula, particularly the contributions from both $i^{\ell_{2}}$ and $\eta_{2}$. As will be seen below, these cancellations are a general pattern when we move to higherorder matrix elements.

Our approximation for the higher-order matrix elements is based on the above formalism. Starting with the third-order element, the equivalent of Eq. (A4) is

$$
\begin{aligned}
M^{(3)}\left(\vec{k}_{3} ; \Omega, \omega\right) \\
=-i\left(\frac{4 \pi}{3}\right)^{3 / 2}(8 \pi)^{3 / 2} \tilde{E}_{\Omega} \tilde{E}_{\omega}^{2} \\
\quad \times \sum_{\ell_{3}}(-i)^{\ell_{3}} e^{i \eta_{3}} Y_{\ell_{3}}\left(\hat{k}_{3}\right) \\
\quad \times \sum_{\ell_{2}, \ell_{1}}\left\langle Y_{\ell_{3}}\left|Y_{1,0}\right| Y_{\ell_{2}}\right\rangle\left\langle Y_{\ell_{2}}\left|Y_{1,0}\right| Y_{\ell_{1}}\right\rangle\left\langle Y_{\ell_{1}}\left|Y_{1,0}\right| Y_{\ell_{i}}\right\rangle \\
\quad \times T_{\ell_{3}, \ell_{2}, \ell_{1}, \ell_{i}}^{(3)}\left(k_{3} ; \Omega, \omega\right) \\
=\tilde{E}_{\Omega} \tilde{E}_{\omega}^{2} \sum_{\ell_{3}} \mathcal{M}_{\ell_{3}}^{(3)}\left(k_{3}\right) Y_{\ell_{3}}\left(\hat{k}_{3}\right) .
\end{aligned}
$$


Here

$$
T_{\ell_{3}, \ell_{2}, \ell_{1}, \ell_{i}}^{(3)}\left(k_{3} ; \Omega, \omega\right)=\left\langle R_{3}|r| \rho_{2}\right\rangle
$$

with the second-order perturbed wave function

$$
\left|\rho_{2}\right\rangle=\int_{0}^{\infty} d \epsilon_{2}^{\prime} \frac{\left|R_{2}^{\prime}\right\rangle\left\langle R_{2}^{\prime}|r| \rho_{1}\right\rangle}{\left(\epsilon_{2}-\epsilon_{2}^{\prime}+i \varepsilon\right)} .
$$

In the asymptotic approximation, we obtain

$$
\rho_{2}(r) \approx-\frac{\pi N_{2}}{r} T^{(2)}\left(k_{2}\right) e^{i\left(k_{2} r+\phi_{2}(r)\right)}
$$

and, consequently,

$$
\begin{aligned}
T_{3}^{(3)}\left(k_{3}\right) \approx & \frac{T^{(2)}\left(k_{2}\right)}{\sqrt{k_{2} k_{3}}} \frac{e^{-Z\left(1 / k_{2}-1 / k_{3}\right) \pi / 2}}{\left(k_{2}-k_{3}\right)^{2}} i^{\ell_{3}-\ell_{2}+1} e^{i\left(\eta_{2}-\eta_{3}\right)} \\
& \times \frac{\left(2 k_{2}\right)^{i Z / k_{2}}}{\left(2 k_{3}\right)^{i Z / k_{3}}} \frac{\left(\Gamma\left[2+i Z\left(1 / k_{2}-1 / k_{3}\right)\right]+\gamma\left(k_{3}, k_{2}\right)\right)}{\left(k_{2}-k_{3}\right)^{i Z\left(1 / k_{2}-1 / k_{3}\right)}} .
\end{aligned}
$$

Looking at the phases,

$$
\begin{aligned}
\arg [ & \left.T_{\ell_{3}, \ell_{2}, \ell_{1}, \ell_{i}}^{(3)}\left(k_{3}\right)\right] \\
& =\frac{\pi}{2}\left(\ell_{3}-\ell_{2}+1\right)+\left(\eta_{2}-\eta_{3}\right)+\phi_{3,2}^{c c}+\arg \left[T^{(2)}\left(k_{2}\right)\right] \\
& =\frac{\pi}{2}\left(\ell_{3}-\ell_{1}+2\right)+\left(\eta_{1}-\eta_{3}\right)+\phi_{3,2}^{c c}+\phi_{2,1}^{c c}, \quad \text { (A20) }
\end{aligned}
$$

and, therefore, since the above radial matrix element is independent of the intermediate angular momentum $\ell_{2}$, we obtain that

$$
\begin{aligned}
\arg \left[\mathcal{M}_{\ell_{3}, \ell_{2}, \ell_{1}, \ell_{i}}^{(3)}\left(k_{3}\right)\right] & =\frac{-\pi}{2}-\frac{\pi \ell_{3}}{2}+\eta_{3}+\arg \left[T^{(3)}\left(k_{3}\right)\right] \\
& =\frac{\pi}{2}-\frac{\pi \ell_{1}}{2}+\eta_{1}+\phi_{3,2}^{c c}+\phi_{2,1}^{c c} .
\end{aligned}
$$

By repeating the procedure, i.e., straightforward induction, the phase of the $N$ th-order matrix element can be written as

$$
\begin{aligned}
\arg \left[\mathcal{M}_{\ell_{N} ; \ell_{1}}^{(N)}\left(k_{N}\right)\right]= & \frac{(N-2) \pi}{2}-\frac{\pi \ell_{1}}{2}+\eta_{\ell_{1}} \\
& +\phi_{k_{2}, k_{1}}^{c c}+\phi_{k_{3}, k_{2}}^{c c}+\cdots+\phi_{k_{N}, k_{N-1}}^{c c} .
\end{aligned}
$$

The analytical form of $\arg \left[\mathcal{M}_{\ell_{N} ; \ell_{1}}^{(N)}\left(k_{N}\right)\right]$ only depends on the angular momenta of the first intermediate state after the XUV step.
[1] P. M. Paul, E. S. Toma, P. Breger, G. Mullot, F. Augé, P. Balcou, H. G. Muller, and P. Agostini, Science 292, 1689 (2001).

[2] K. Klünder, J. M. Dahlström, M. Gisselbrecht, T. Fordell, M. Swoboda, D. Guénot, P. Johnsson, J. Caillat, J. Mauritsson, A. Maquet, R. Taïeb, and A. L'Huillier, Phys. Rev. Lett. 106, 143002 (2011).

[3] M. Isinger, R. J. Squibb, D. Busto, S. Zhong, A. Harth, D. Kroon, S. Nandi, C. L. Arnold, M. Miranda, J. M. Dahlström, E. Lindroth, R. Feifel, M. Gisselbrecht, and A. L'Huillier, Science 358, 893 (2017).

[4] D. Guénot, K. Klünder, C. L. Arnold, D. Kroon, J. M. Dahlström, M. Miranda, T. Fordell, M. Gisselbrecht, P. Johnsson, J. Mauritsson, E. Lindroth, A. Maquet, R. Taïeb, A. L'Huillier, and A. S. Kheifets, Phys. Rev. A 85, 053424 (2012).

[5] A. W. Bray, F. Naseem, and A. S. Kheifets, Phys. Rev. A 97, 063404 (2018).

[6] W. Boutu, S. Haessler, H. Merdji, P. Breger, G. Waters, M. Stankiewicz, L. J. Frasinski, R. Taieb, J. Caillat, A. Maquet, P. Monchicourt, B. Carre, and P. Salieres, Nat. Phys. 4, 545 (2008).

[7] S. Haessler, J. Caillat, W. Boutu, C. Giovanetti-Teixeira, T. Ruchon, T. Auguste, Z. Diveki, P. Breger, A. Maquet, B. Carré, R. Taïeb, and P. Salières, Nat. Phys. 6, 200 (2010).

[8] R. Locher, L. Castiglioni, M. Lucchini, M. Greif, L. Gallmann, J. Osterwalder, M. Hengsberger, and U. Keller, Optica 2, 405 (2015).

[9] M. Lucchini, L. Castiglioni, L. Kasmi, P. Kliuiev, A. Ludwig, M. Greif, J. Osterwalder, M. Hengsberger, L. Gallmann, and U. Keller, Phys. Rev. Lett. 115, 137401 (2015).
[10] M. J. Ambrosio and U. Thumm, Phys. Rev. A 94, 063424 (2016).

[11] J. Dahlström, D. Guénot, K. Klünder, M. Gisselbrecht, J. Mauritsson, A. L'Huillier, A. Maquet, and R. Taïeb, Chem. Phys. 414, 53 (2013).

[12] I. A. Ivanov, J. M. Dahlström, E. Lindroth, and A. S. Kheifets, arXiv:1605.04539.

[13] J. Fuchs, N. Douguet, S. Donsa, F. Martin, J. Burgdörfer, L. Argenti, L. Cattaneo, and U. Keller, Optica 7, 154 (2020).

[14] M. Swoboda, J. M. Dahlström, T. Ruchon, P. Johnsson, J. Mauritsson, A. L'Huillier, and K. J. Schafer, Laser Phys. 19, 1591 (2009).

[15] P. K. Marojuet et al., Nature 578, 386 (2020).

[16] A. Harth, N. Douguet, K. Bartschat, R. Moshammer, and T. Pfeifer, Phys. Rev. A 99, 023410 (2019).

[17] J. M. Dahlström, A. L'Huillier, and A. Maquet, J. Phys. B: At. Mol. Opt. Phys. 45, 183001 (2012).

[18] F. H. M. Faisal, Theory of Multiphoton Processes, Physics of Atoms and Molecules (Springer-Verlag, Berlin, 1987).

[19] P. Lambropoulos, in Advances in Atomic and Molecular Physics, edited by D. R. Bates and B. Bederson (Academic Press, 1976), Vol. 12, pp. 87-164.

[20] L. A. A. Nikolopoulos, Phys. Rev. A 73, 043408 (2006).

[21] N. Douguet, A. N. Grum-Grzhimailo, E. V. Gryzlova, E. I. Staroselskaya, J. Venzke, and K. Bartschat, Phys. Rev. A 93, 033402 (2016).

[22] K. R. Hamilton, T. Pauly, K. Bartschat, N. Douguet, D. Bharti, and A. Harth, Conference: 51st Annual Meeting of the Division of Atomic, Molecular, and Optical Physics of the American Physical Society, Contribution: K01.00048, June 2020. 